\title{
Epidemics and immunization in scale-free networks
}

\author{
R. Pastor-Satorras and A. Vespignani
}

WILEY-VCH Verlag Berlin GmbH

October 22, 2018 



\title{
1 Epidemics and immunization in scale-free networks
}

\author{
Romualdo Pastor-Satorras \\ Departament de Física i Enginyeria Nuclear \\ Universitat Politècnica de Catalunya \\ 08034 Barcelona, Spain
}

Alessandro Vespignani

The Abdus Salam International Centre for Theoretical Physics (ICTP)

P.O. Box 586, 34100 Trieste, Italy

\subsection{Introduction}

Epidemic models are heavily affected by the connectivity patterns characterizing the population in which the infective agent spreads [1, 2, 3]. Many models map this pattern in terms of networks, in which nodes represent individuals and links represent the possible contacts along which the epidemic diffuses. In this perspective scale-free (SF) networks [ 4 , 5, 6] represents a very interesting cases since they exhibit a power-law connectivity distribution

$$
P(k) \sim k^{-\gamma}
$$

for the probability $P(k)$ that a node of the network has $k$ connections to other nodes. For connectivity exponents in the range $2<\gamma \leq 3$ this fact implies that each node (element of the population) has a statistically significant probability of having a very large number of connections compared to the average connectivity $\langle k\rangle$ of the network. In mathematical terms, the implicit divergence of $\left\langle k^{2}\right\rangle$ is signalling the extreme heterogeneity of the connectivity pattern, and it easy to foresee that this property is going to change drastically the behavior of epidemic outbreaks in SF networks. The interest in the study of epidemic models in SF networks is enhanced by the evidence that both the Internet [7, 8, 9, 10, 11] and the maps of human sexual contacts [12] are characterized by scale-free connectivity properties. The Internet and the web of sexual contacts are, in fact, the natural environment in which cyber viruses and sexually transmitted diseases (STD), respectively, live and proliferate. The study and characterization of epidemics in scale-free networks is therefore of potential importance for those seeking to control and arrest human and electronic plagues. These considerations motivate the analysis of the effect of complex network topologies in standard epidemic models leading to several interesting and novel results [13, 14, 15,.

Perhaps, the most surprising result, first originated by the inspection of the susceptibleinfected-susceptible (SIS) model, is that the spread of infections is tremendously strengthened on SF networks [15, 16]. Opposite to standard models, epidemic processes in these 
networks do not possess any epidemic threshold below which the infection cannot produce a major epidemic outbreak or an endemic state. In principle, SF networks are prone to the persistence of diseases whatever infective rate they may have. The same peculiar absence of an epidemic threshold has been readily confirmed in other epidemic models such as the susceptible-infected-removed (SIR) model [17, 18, 19] and appears as a general feature of epidemic spreading in SF networks. This feature reverberates also in the choice of immunization strategies [20, 21] and changes radically the standard epidemiological framework usually adopted in the description and characterization of disease propagation.

In this chapter we want to provide a review of the main results obtained in the modeling of epidemic spreading in SF networks. In particular, we want to show the different epidemiological framework originated by the lack of any epidemic threshold and how this feature is rooted in the extreme heterogeneity of the SF networks' connectivity pattern. As a real world example of epidemic spreading occurring on SF connectivity patterns, we shall consider the diffusion of computer viruses. Computer virus spreading, in fact, can be characterized by simple population models that do not consider properties such as gender, sex, or age, that must be included in the modeling of STD and other kinds of epidemics. On the other hand, computer viruses proliferate in the Internet, that is a capital example of SF network, and it is natural to include this topology in their modeling. Finally, many real data are available in computers epidemiology and we can use them to show experimentally the failure of the standard epidemic framework and support the new picture arising for SF networks.

We also present how the scale-free nature of the network calls for different immunization strategies in order to eradicate infections. Opposite to standard models, it is found that SF networks do not acquire global immunity from major epidemic outbreaks even in the presence of unrealistically high densities of randomly immunized individuals. Successful immunization strategies, therefore, can be developed only by taking advantage of the inhomogeneous connectivity properties of the scale-free connectivity patterns. Finally we consider the effect of the network finite size, referring to real systems which are actually made up by a finite number of individuals. The presented results provide a general view of the novel features of epidemic models in SF networks that, besides the application to computer viruses, prompt to the relevant implications of these studies in human and animal epidemiology.

\subsection{Computers and epidemiology}

In a classic paper [22], it is described the Domain-Name-Server (DNS) cache corruption spreading as a natural computer virus proliferating on the Internet. Computers on the Internet rely upon DNS servers to translate Internet protocol addresses into computer names and vice-versa. On their turn, DNS servers communicate with their DNS peers to share and update these informations. The updating is periodic in time and in the meanwhile, translation tables are "cached" and eventually transmitted to the other DNS peers. If any portion of this cache is corrupted, the DNS server will provide incorrect addresses not only to requesting computers but to DNS peers as well, propagating the error. At the same time, any DNS server can get "cured" by an updating with an error-free DNS peer. The same kind of processes can occur with routing tables exchanged by routers. This propagation of errors occurring on routers and servers that are physically linked is a typical example of epidemic process, in which the 
corruption (virus) is transmitted from infected to healthy individuals.

From a more familiar point of view, however, computer viruses are usually referred to as little programs that can reproduce themselves by infecting other programs [23, 24]. The basic mechanism of infection is as follows: When the virus is active inside the computer, it is able to copy itself, by different ways, into the code of other, clean, programs. When the newly infected program is run into another computer, the code of the virus is executed first, becoming active and being able to infect other programs. Apart from reproducing themselves, computer viruses perform threatening tasks that range from flashing innocuous messages on the screen to seriously corrupt data stored in the computer. These deleterious effects render most computer viruses as dangerous as their biological homonyms, and explain the interest, both commercial and scientific, arisen around their study.

Computer viruses have evolved in time (driven of course by their programmers' skills), adopting different strategies that take advantage of the different weak points of computers and software. Computer viruses can be classified into three main classes, or strains [24]. The first strain includes file viruses that infect application programs. A second and more harming family contains the boot-sector viruses that infect the boot sector of floppy disks and hard drives, a portion of the disk containing a small program in charge of loading the operating system of the computer. A third and nowadays prevailing strain is formed by the macro viruses. These viruses are independent of the platform's hardware and infect data files, such as documents produced with spreadsheets or word processors. They are coded using the macro instructions that are appended in the document, instructions used to perform a set of automatic actions, such as formatting the documents or typing long sequences of characters. In addition, with the ever more efficient deployment of antivirus software, more harmful viruses combining together the properties of the main strains have been developed.

Noticeably, however, the nowadays dominant and most aggressive type of cyber organisms is represented by the worms family. Worms are actually viruses infecting the computer with mechanisms similar to usual viruses and making a particularly effective use of the e-mail for infecting new computers. In fact, by using the instructions of some commercial mail software applications, worms are capable of sending themselves to all the e-addresses found in the address-book of the person receiving the infected mail. This possibility renders worms the most effective viruses, especially in terms of the velocity at which they can propagate starting from a single infection.

The spreading of computer viruses has been studied for long years, in close analogy with the models developed for the study of the transmission of biological diseases (for a review see Refs. [25, 26]). In this biological framework, the key point is the description of the epidemic process in terms of individuals and their interactions. In this simplified formalism, individuals can only exist in a discrete set of states, such as susceptible (or healthy), infected (and ready to spread the disease), immune, dead (or removed), etc. On the other hand, the interactions among individuals are schematized in the structure of the contacts along which the epidemics can propagate. Within this formalism, the system can be described as a network or graph [27], in which the nodes represent the individuals and the links are the connections along which the epidemics propagates.

Standard epidemiological models usually consider homogeneous networks, which are those that have a connectivity distribution peaked at an average connectivity $\langle k\rangle$, and decaying exponentially fast for $k \ll\langle k\rangle$ and $k \gg\langle k\rangle$. A typical example of deterministic homogeneous 
network is the standard hypercubic lattice, while among the random homogeneous network we can count the Erdös-Rényi model [28] and the Watts-Strogatz model [29]. On the other hand, as we shall see in the following, computer viruses and worms spread in environments characterized by scale-free connectivities. This will lead to the failure of the standard epidemic picture and will naturally introduce the scale-free connectivity as an essential ingredient for the understanding of computer viruses.

\subsection{Epidemic spreading in homogeneous networks}

The simplest epidemiological model one can consider is the susceptible-infected-susceptible (SIS) model [2, 3]. In the SIS model, individuals can only exist in two discrete states, namely, susceptible and infected. These states completely neglect the details of the infection mechanism within each individual. The disease transmission is also described in an effective way. At each time step, each susceptible node is infected with probability $\nu$ if it is connected to one or more infected nodes. At the same time, infected nodes are cured and become again susceptible with probability $\delta$, defining an effective spreading rate

$$
\lambda=\frac{\nu}{\delta} .
$$

Without lack of generality, we set can set $\delta=1$, since it only affects at the definition of the time scale of the disease propagation. Individuals thus run stochastically through the cycle

$$
\text { susceptible } \rightarrow \text { infected } \rightarrow \text { susceptible, }
$$

and hence the name of the model. The SIS model does not take into account the possibility of individuals removal due to death or acquired immunization which would lead to the socalled susceptible-infected-removed (SIR) model [2, 3]. It is mainly used as a paradigmatic model for the study of infectious disease leading to an endemic state with a stationary and constant value for the density of infected individuals, i.e. the degree to which the infection is widespread in the population. The SIS has been adopted in the modeling of computer viruses and worms since, also in the presence of antiviruses, computer immunization statistically depends upon the user concerns in not skipping the antivirus control when opening e-mail attachments or new files.

The analytical study of the SIS model can be undertaken in terms of a dynamical meanfield (MF) theory. For homogeneous networks, in which the connectivity fluctuations are very small, we can approach the MF theory by means of a reaction equation for the total prevalence $\rho(t)$, defined as the density of infected nodes present at time $t$. That is, we can consider all the nodes as equivalent, irrespective of their corresponding connectivity. The reaction equation for $\rho(t)$ can be written as

$$
\partial_{t} \rho(t)=-\rho(t)+\lambda\langle k\rangle \rho(t)[1-\rho(t)] .
$$

The MF character of this equation stems from the fact that we have neglected the density correlations among the different nodes. In Eq. (1.3) we have also ignored all higher order corrections in $\rho(t)$, since we are interested in the onset of the infection close to the point $\rho(t) \ll 1$. The first term on the right-hand-side in Eq. (1.3) considers infected nodes becoming 


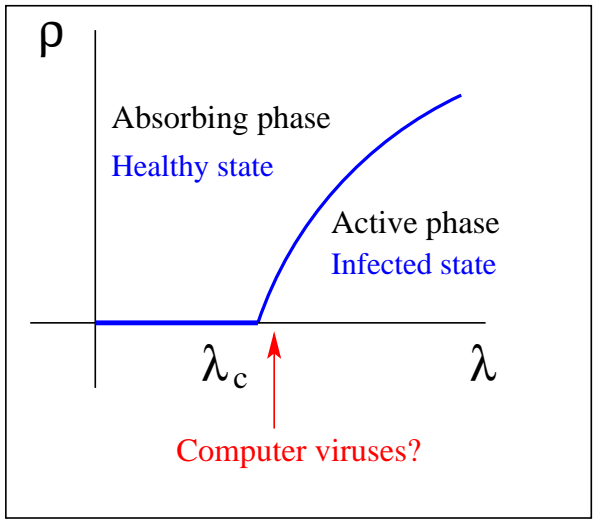

Figure 1.1: Schematic phase diagram for the SIS model in homogeneous networks. The epidemic threshold $\lambda_{c}$ separates an active or infected phase, with finite prevalence, from an absorbing or healthy phase, with null prevalence. The very small prevalence and long lifetimes observed in computer virus data are only compatible with a value of $\lambda$ infinitesimally close to the epidemic threshold.

healthy with unit rate. The second term represents the average density of newly infected nodes generated by each active node. This is proportional to the infection spreading rate, $\lambda$, to the number of links emanating from each node, and to the probability that a given link points to a healthy node, $[1-\rho(t)]$. In the homogeneous networks we are considering here, connectivity has only very small fluctuations $\left(\left\langle k^{2}\right\rangle \sim\langle k\rangle\right)$ and as a first approximation we have considered that each node has the same number of links, $k \simeq\langle k\rangle$. This is equivalent to an homogeneity assumption for the system's connectivity. In writing this last term of the equation we are also assuming the homogeneous mixing hypothesis [3], which asserts that the force of the infection (the per capita rate of acquisition of the disease for the susceptible individuals) is proportional to the density of infected individuals $\rho(t)$. The homogeneous mixing hypothesis is indeed equivalent to a mean-field treatment of the model, in which one assumes that the rate of contacts between infectious and susceptibles is constant, and independent of any possible source of heterogeneity present in the system. Another implicit assumption of this model is that the time scale of the disease is much smaller than the lifespan of individuals; therefore we do not include in the equations terms accounting for the birth or natural death of individuals.

After imposing the stationarity condition $\partial_{t} \rho(t)=0$, we obtain the equation, valid for the behavior of the system at large times,

$$
\rho[-1+\lambda\langle k\rangle(1-\rho)]=0
$$

for the steady state density $\rho$ of infected nodes. This equation defines an epidemic threshold $\lambda_{c}=\langle k\rangle^{-1}$, and yields:

$$
\begin{array}{ll}
\rho=0 & \text { if } \lambda<\lambda_{c} \\
\rho=\left(\lambda-\lambda_{c}\right) / \lambda & \text { if } \lambda \geq \lambda_{c} .
\end{array}
$$


The most significant prediction of this model is the presence of a nonzero epidemic threshold $\lambda_{c}$ [30, 21. If the value of $\lambda$ is above the threshold, $\lambda \geq \lambda_{c}$, the infection spreads and becomes persistent. Below the threshold, $\lambda<\lambda_{c}$, the infection dies exponentially fast. From the point of view of nonequilibrium phase transitions, the SIS model exhibits an absorbing-state phase transition [30] at the threshold $\lambda_{c}$, separating an active or infected phase, with finite prevalence, from an absorbing or healthy phase, with null prevalence. A qualitative picture of the phase diagram of this transition is depicted in Figure 1.1. It is easy to recognize that the SIS model is a generalization of the contact process model, widely studied in this context as the paradigmatic example of an absorbing-state phase transitions to a unique absorbing state [30].

To summarize, the main prediction of the SIS model in homogeneous networks is the presence of a positive epidemic threshold, proportional to the inverse of the average number of neighbors of every node, $\langle k\rangle$, below which the epidemics always dies, and endemic states are impossible.

\subsection{Real data analysis}

The statistical properties of computer virus data have been analyzed by several authors, in close analogy with the classical epidemiology of biological diseases [1], 2, 3]. Within this framework, studies have focused specially in the measurement of the virus prevalence, defined as the average fraction of computers infected with respect to the total number of computers present. From these studies [24, 31, 26] two main conclusions have been drawn. First, viruses which are able to survive in order to produce a significant outbreak usually reach an endemic or metastable steady state, with a stationary prevalence. The second empirical observation is that these endemic viruses do attain in general a very small average prevalence, that can be of the order of one out of 1000 computers or less.

More recently, other studies [15] have focused in the dynamics of the spreading process, measuring the surviving probability of homogeneous groups of viruses, classified according to their infection mechanism (strains). In these studies one considers the total number of viruses of a given strain that are born and die within a given observation window. The surviving probability $P_{s}(t)$ of the strain is defined as the fraction of viruses still alive at time $t$ after their birth. Figure 1.2 reproduces the results reported in Ref. [15], obtained from prevalence data from the Virus Bulletin $\rrbracket$ in the period February 1996 to March 2000, covering a time interval of 50 months.

Figure 1.2 shows that the surviving probability suffers a sharp drop in the first two months of a virus' life. On the other hand, Figure 1.2 also shows for larger times a clean exponential tail,

$$
P_{s}(t) \sim \exp (-t / \tau)
$$

where $\tau$ represents the characteristic life-time of the virus strain. The numerical fit of the data [15. yields $\tau \simeq 14$ months for boot and macro viruses and $\tau \simeq 6-9$ months for file viruses.

\footnotetext{
${ }^{1}$ Virus prevalence data publicly available at the web site http://www. virusbtn.com.
} 


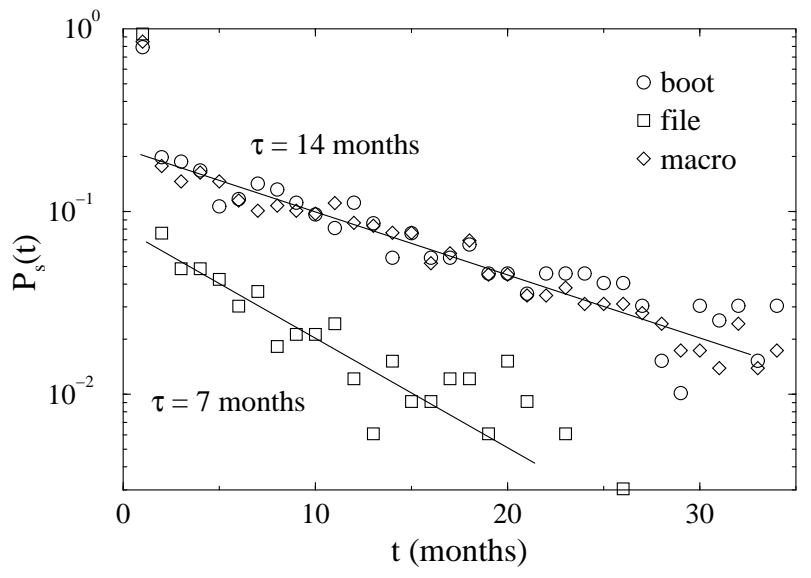

Figure 1.2: Surviving probability for the three main strands of computer viruses. After a sharp initial drop, it is clear the presence of an exponential decay, with an associated characteristic time $\tau$ that depends on the given strand.

When comparing the theoretical picture delivered by the SIS model on homogeneous networks with the behavior observed in real computer viruses, one is faced with an unexpected and paradoxical conclusion. First of all, the extremely low prevalence shown by endemic viruses is only compatible with the phase diagram sketched in Figure 1.1 in the very unlikely chance that all surviving viruses are constructed such that their respective spreading rate $\lambda$ is tuned infinitesimally close to $\lambda_{c}$, above the epidemic threshold. On the other hand, the characteristic life times observed in the analysis of the surviving probability of the different virus strains are impressively large if compared with the interval in which anti-virus software is available on the market (usually within days or weeks after the first incident report) and corresponds to the occurrence of metastable endemic states. Such a long lifetime on the scale of the typical spread/recovery rates would suggest an effective spreading rate larger than the epidemic threshold, which is in contradiction with the always low prevalence levels of computer viruses but in the case of an unrealistic tuning of all viruses to the system epidemic threshold. In summary, the comparison with the known experimental data points out that the view obtained so far with the modeling of computer viruses is very instructive, but fails to represent, even at a qualitative level, the nature of the real phenomenon. The explanation of this discrepancy has been claimed to be one of the most important open problems in computer virus epidemiology [32].

The key point to elucidating the riddle posed by computer viruses resides in the capacity of many of them to propagate via data exchange with communication protocols (FTP, e-mails, etc.) [24]. Viruses will spread preferentially to computers which are highly connected to the outer world and are thus proportionally exchanging more data and information. It is thus rather intuitive to consider the scale-free Internet connectivity as the effective one on which the spreading occurs. For instance, this is the case of natural computer viruses which spread on the topology identified by routers and servers [7, 8, 9p. Apart from the Internet, scale-free 
properties emerge also in the world-wide-web [4, 33] and in social networks [34]. The fact that all virus strains show the same statistical features indicates that very likely all of them spread on scale-free connectivity patterns. Further and strong support for this conclusion comes from the recent study of a social network of e-mail exchange within a community of users [35], which was proven to have a scale-free connectivity, with an exponent close to 2 . This finding has an immediate repercussion on the modeling of worms, whose spreading environment is in fact given by this kind of network.

The conclusion from the above arguments is that computer viruses spread in a scale-free network, in which, even though the average connectivity is well defined, the connectivity fluctuations are unbounded; i.e. there is always a finite probability that a node has a number of neighbors much larger than the average value. These fluctuations in the connectivity are the key difference with respect to the epidemic models discussed in homogeneous graphs, and they must be included in a correct characterization of the system.

\subsection{Epidemic spreading in scale-free networks}

In order to fully take into account connectivity fluctuations in a analytical description of the SIS model, we have to relax the homogeneity assumption used for homogeneous networks, and work instead with the relative density $\rho_{k}(t)$ of infected nodes with given connectivity $k$; i.e. the probability that a node with $k$ links is infected. The dynamical mean-field equations can thus be written as [15, 16]

$$
\frac{d \rho_{k}(t)}{d t}=-\rho_{k}(t)+\lambda k\left[1-\rho_{k}(t)\right] \Theta\left[\left\{\rho_{k}(t)\right\}\right],
$$

where also in this case we have considered a unitary recovery rate and neglected higher order terms $\left(\rho_{k}(t) \ll 1\right)$. The creation term considers the probability that a node with $k$ links is healthy $\left[1-\rho_{k}(t)\right]$ and gets the infection via a connected node. The probability of this last event is proportional to the infection rate $\lambda$, the real number of connections $k$, and the probability $\Theta\left[\left\{\rho_{k}(t)\right\}\right]$ that any given link points to an infected node. We make the assumption that $\Theta$ is a function of the partial densities of infected nodes $\left\{\rho_{k}(t)\right\}$. In the steady (endemic) state, the $\rho_{k}$ are functions of $\lambda$. Thus, the probability $\Theta$ becomes also an implicit function of the spreading rate, and by imposing the stationarity condition $\partial_{t} \rho_{k}(t)=0$, we obtain

$$
\rho_{k}=\frac{k \lambda \Theta(\lambda)}{1+k \lambda \Theta(\lambda)} .
$$

This set of equations show that the higher the node connectivity, the higher the probability to be in an infected state. This inhomogeneity must be taken into account in the computation of $\Theta(\lambda)$. The exact calculation of $\Theta$ for a general network is a very difficult task. However, we can exactly compute its value for the case of a random SF network, in which there are no correlations among the connectivities of the different nodes [15, 16]. Indeed, the probability that a link points to a node with $s$ connections is equal to $s P(s) /\langle k\rangle$, which yields an average probability of a link pointing to an infected node

$$
\Theta(\lambda)=\frac{1}{\langle k\rangle} \sum_{k} k P(k) \rho_{k}
$$


Since $\rho_{k}$ is on its turn a function of $\Theta(\lambda)$, we obtain a self-consistency equation that allows to find $\Theta(\lambda)$ and an explicit form for Eq. (1.9). Finally, we can evaluate the order parameter (persistence) $\rho$ using the relation

$$
\rho=\sum_{k} P(k) \rho_{k} .
$$

The self-consistent Eqs. (1.9) and (1.10) can be approximately solved, in the limit of small $\Theta$, for any scale-free connectivity distribution [16]. However, we can very easily calculate the epidemic threshold by just noticing that $\lambda_{c}$ is the value of $\lambda$ above which it is possible to obtain a nonzero solution for $\Theta$. In fact, from Eqs. (1.9) and (1.10), we obtain the selfconsistent relation

$$
\Theta=\frac{1}{\langle k\rangle} \sum_{k} k P(k) \frac{\lambda k \Theta}{1+\lambda k \Theta},
$$

where $\Theta$ is now a function of $\lambda$ alone [15, 16. The solution $\Theta=0$ is always satisfying the consistency equation. A non-zero stationary prevalence $\left(\rho_{k} \neq 0\right)$ is obtained when the righthand-side and the left-hand-side of Eq. (1.12), expressed as function of $\Theta$, cross in the interval $0<\Theta \leq 1$, allowing a nontrivial solution. It is easy to realize that this corresponds to the inequality

$$
\left.\frac{d}{d \Theta}\left(\frac{1}{\langle k\rangle} \sum_{k} k P(k) \frac{\lambda k \Theta}{1+\lambda k \Theta}\right)\right|_{\Theta=0} \geq 1
$$

being satisfied. The value of $\lambda$ yielding the equality in Eq. (1.13) defines the critical epidemic threshold $\lambda_{c}$, that is given by

$$
\frac{\sum_{k} k P(k) \lambda_{c} k}{\langle k\rangle}=\frac{\left\langle k^{2}\right\rangle}{\langle k\rangle} \lambda_{c}=1 \quad \Rightarrow \quad \lambda_{c}=\frac{\langle k\rangle}{\left\langle k^{2}\right\rangle} .
$$

This results implies that in SF networks with connectivity exponent $2<\gamma \leq 3$, for which $\left\langle k^{2}\right\rangle \rightarrow \infty$ in the limit of a network of infinite size, we have $\lambda_{c}=0$.

\subsubsection{Analytic solution for the Barabási-Albert network}

In order to discuss in detail a specific example, it is simpler to consider a toy model of SF network, which is easy to generate for simulation purposes and shows the correct connectivity properties. The paradigmatic example of SF network is the Barabási and Albert (BA) model [4. 36. 5]. The construction of the BA graph starts from a small number $m_{0}$ of disconnected nodes; every time step a new vertex is added, with $m$ links that are connected to an old node $i$ with probability

$$
\Pi\left(k_{i}\right)=\frac{k_{i}}{\sum_{j} k_{j}},
$$


where $k_{i}$ is the connectivity of the $i$-th node. This algorithm implements the so-called "richget-richer" paradigm [ 4 ], that implies that highly connected nodes have always larger chances to become even more connected. The networks generated this way have a connectivity distribution $P(k) \sim k^{-3}$.

In the explicit calculations for the BA model, we use a continuous $k$ approximation that allows the practical substitution of series with integrals [\#. The full connectivity distribution is thus given by $P(k)=2 m^{2} k^{-3}$. By noticing that the average connectivity is $\langle k\rangle=\int_{m}^{\infty} k P(k) d k=2 m$, Eq. (1.10) gives

$$
\Theta(\lambda)=m \lambda \Theta(\lambda) \int_{m}^{\infty} \frac{1}{k} \frac{d k}{1+k \lambda \Theta(\lambda)}=m \lambda \Theta(\lambda) \log \left(1+\frac{1}{m \lambda \Theta(\lambda)}\right),
$$

which yields the solution

$$
\Theta(\lambda)=\frac{e^{-1 / m \lambda}}{\lambda m}\left(1-e^{-1 / m \lambda}\right)^{-1}
$$

In order to find the behavior of the density of infected nodes we have to solve Eq. (1.11), that reads as

$$
\rho=2 m^{2} \lambda \Theta(\lambda) \int_{m}^{\infty} \frac{1}{k^{2}} \frac{d k}{1+k \lambda \Theta(\lambda)}=2 m^{2} \lambda \Theta(\lambda)\left[\frac{1}{m}+\lambda \Theta(\lambda) \log \left(1+\frac{1}{m \lambda \Theta(\lambda)}\right)\right] .
$$

By substituting the obtained expression for $\Theta(\lambda)$ we find at lowest order in $\lambda$

$$
\rho \sim 2 e^{-1 / m \lambda}
$$

This result shows the absence of any epidemic threshold or critical point in the model; i.e., $\lambda_{c}=0$, in agreement with the result from Eq. (1.14) for a scale-free network with $\left\langle k^{2}\right\rangle=\infty$. Numerical simulations of the SIS model performed on a BA network confirm the analytical picture extracted from the mean-field analysis. Figure 1.3 shows the total prevalence $\rho$ in the steady state as a function of the spreading rate $\lambda[16]$. As we can observe, it approaches zero in a continuous and smooth way, compatible with the presence of a vanishing epidemic threshold (see for comparison the behavior expected for a homogeneous network, also drawn in Figure 1.3. . On the other hand, Figure 1.4 represents $\rho$ in a semilogarithmic plot as a function of $1 / \lambda$, which shows that $\rho \sim \exp (-C / \lambda)$, where $C$ is a constant independent of the size $N$ of the network.

The spreading dynamical properties of the model can also be studied by means of numerical simulations [16]. For example, the surviving probability $P_{s}(t)$ for a fixed value of $\lambda$ and different network sizes $N$ is represented in Figure 1.5. In this case, we recover an exponential behavior in time, that has its origin in the finite size of the network. In fact, for any finite system, the epidemic will eventually die out because there is a finite probability that all individuals cure the infection at the same time. This probability is decreasing with the system size and the lifetime is infinite only in the thermodynamic limit $N \rightarrow \infty$. However, the lifetime becomes virtually infinite (the metastable state has a lifetime too long for our observation window) for large enough sizes that depend upon the spreading rate $\lambda$. This is a well-known 


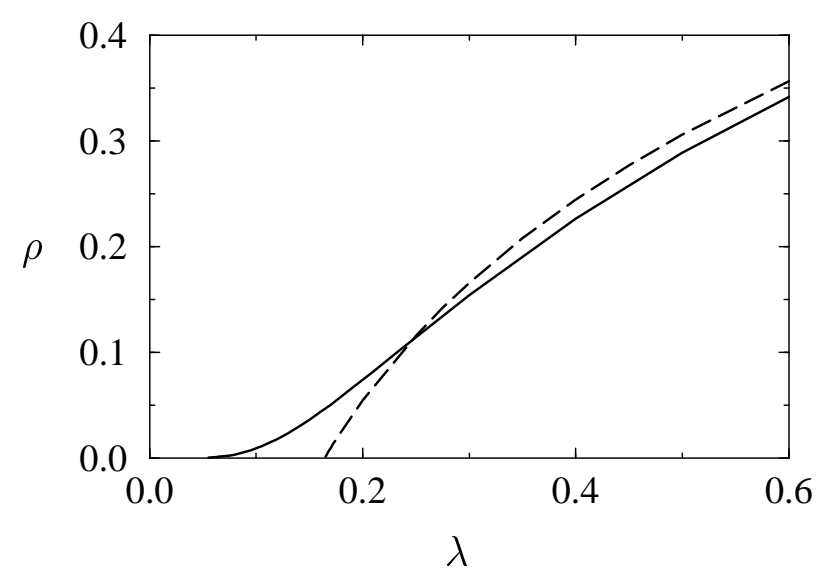

Figure 1.3: Total prevalence $\rho$ for the SIS model in a BA network (full line) as a function of the spreading rate $\lambda$, compared with the theoretical prediction for a homogeneous network (dashed line).

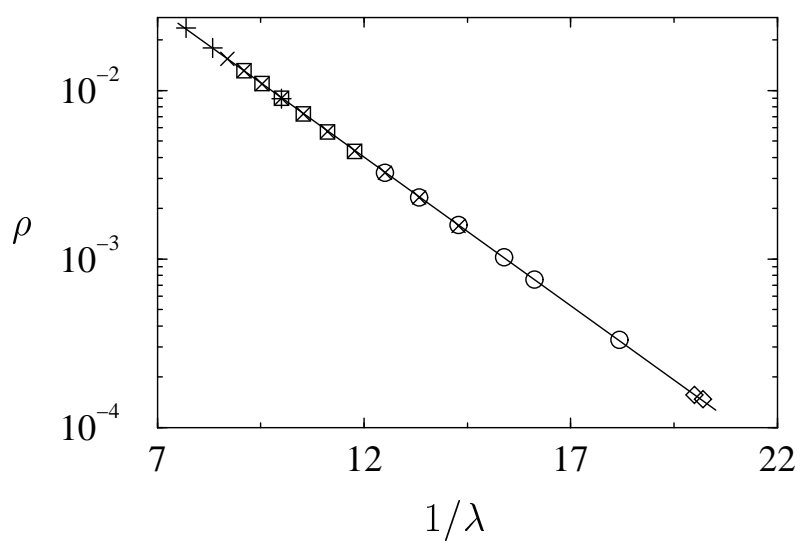

Figure 1.4: Persistence $\rho$ as a function of $1 / \lambda$ for BA networks of different sizes: $N=10^{5}$ $(+), N=5 \times 10^{5}(\square), N=10^{6}(\times), N=5 \times 10^{6}(\circ)$, and $N=8.5 \times 10^{6}(\diamond)$. The linear behavior on the semi-logarithmic scale proves the stretched exponential behavior predicted for the persistence. The full line is a fit to the form $\rho \sim \exp (-C / \lambda)$.

feature of the survival probability in finite size absorbing-state systems poised above the critical point [30]. In our case, this picture is confirmed by numerical simulations that show that the average lifetime of the survival probability is increasing with the network size for all the values of $\lambda$.

The outcome of the analysis presented in this section is that the SIS model in a BA scalefree network, with connectivity distribution $P(k) \sim k^{-\gamma}$ and connectivity exponent $\gamma=3$, yields the absence of any epidemic threshold or critical point, $\lambda_{c}=0$. It is worth remarking 


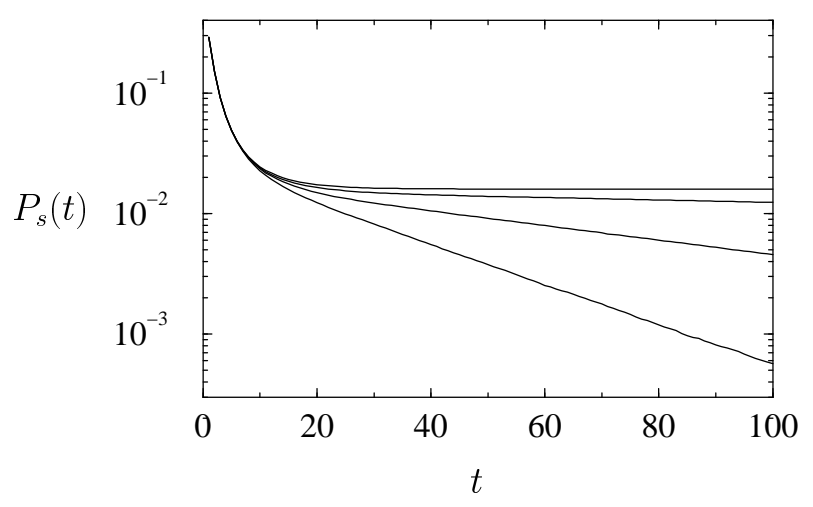

Figure 1.5: Surviving probability $P_{s}(t)$ as a function of time in supercritical spreading experiments in the BA network. Spreading rate $\lambda=0.065$. Network sizes ranging from $N=6.25 \times 10^{3}$ to $N=5 \times 10^{5}$ (bottom to top).

that the present framework can be generalized to networks with $2<\gamma \leq 3$, recovering qualitatively the same results [16]. Only for $\gamma>4$, epidemics on SF networks have the same properties as on homogeneous networks. The emerging picture for epidemic spreading in scale-free networks emphasizes the role of topology in epidemic modeling. In particular, the absence of epidemic threshold and the associated critical behavior in a wide range of scale-free networks provide an unexpected result that radically changes many standard conclusions on epidemic spreading. This indicates that infections can proliferate on these scale-free networks whatever spreading rates they may have. These very bad news are, however, balanced by the exponentially small prevalence for a wide range of spreading rates $(\lambda \ll 1)$. This picture fits perfectly with the observations from real data, and solve the long-standing mystery of the generalized low prevalence of computer viruses without assuming any global tuning of the spreading rates. In addition, the model explains successfully the exponential time decay of the virus surviving probability, with an average lifetime of viral strains that appears to be related to an effective spreading rate and the network size.

\subsubsection{Finite size scale-free networks}

Real systems are actually made up by a finite number of individuals which is far from the thermodynamic limit. This finite population introduces a maximum connectivity $k_{c}$, depending on $N$, which has the effect of restoring a bound in the connectivity fluctuations, inducing in this way an effective nonzero threshold. More generally, we can consider bounded scale-free networks in which the connectivity distribution has the form $P(k) \sim k^{-\gamma} f\left(k / k_{c}\right)$, where the function $f(x)$ decreases very rapidly for $x>1$ [37, 38]. The cut-off $k_{c}$ can be due to the finite size of the network or to the presence of constraints limiting the addition of new links in an otherwise infinite networks. In both cases, $\left\langle k^{2}\right\rangle$ assumes a finite value in bounded SF networks, defining from Eq. (1.14) an effective nonzero threshold due to finite size effects as usually encountered in nonequilibrium phase transitions [30]. This epidemic threshold, however, is not an intrinsic quantity as in homogeneous systems and it vanishes for a increasing 


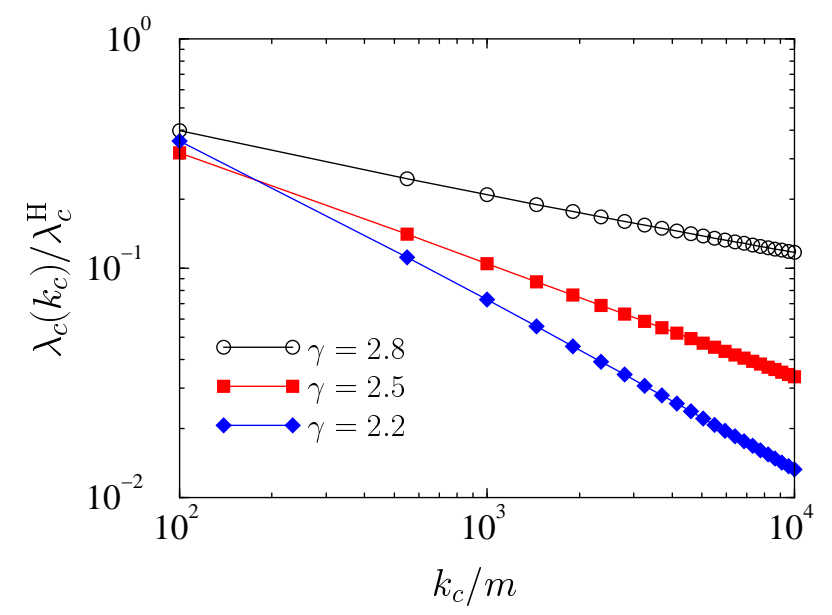

Figure 1.6: Ratio between the effective epidemic threshold in bounded SF networks with exponential cut-off $k_{c}$ and the intrinsic epidemic threshold of homogeneous networks with the same average connectivity, for different values of $\gamma$.

network size or connectivity cut-off. Explicit calculations can be performed for the SIS model [39] in SF networks with exponentially bounded connectivity, $P(k) \sim k^{-\gamma} \exp \left(-k / k_{c}\right)$, obtaining that the effective nonzero epidemic threshold $\lambda_{c}\left(k_{c}\right)$ induced by the cut-off $k_{c}$ behaves as

$$
\lambda_{c}\left(k_{c}\right) \simeq\left(k_{c} / m\right)^{\gamma-3}
$$

where $m$ is the smallest connectivity in the graph. The limit $\gamma \rightarrow 3$, on the other hand, corresponds to a logarithmic divergence, yielding at leading order $\lambda_{c}\left(k_{c}\right) \simeq\left(m \ln \left(k_{c} / m\right)\right)^{-1}$. In all cases we have that the epidemic threshold vanishes when increasing the characteristic cut-off. It is thus interesting to compare the intrinsic epidemic threshold obtained in homogeneous networks with negligible fluctuations and the nonzero effective threshold of bounded SF networks. The intrinsic epidemic threshold of homogeneous networks with constant node connectivity $\langle k\rangle$ is given by $\lambda_{c}^{\mathrm{H}}=\langle k\rangle^{-1}$ [2]. In Fig. 1.6 we report the ratio obtained by using the full expression for $\lambda_{c}\left(k_{c}\right)$. It is striking to observe that, even with relatively small cut-offs $\left(k_{c} \sim 10^{2}-10^{3}\right)$, for $\gamma \approx 2.5$ the effective epidemic threshold of finite size SF networks is smaller by a factor close to $1 / 10$ than the intrinsic threshold obtained on homogeneous networks. This implies that the SF networks weakness to epidemic agents is also present in finite-size and connectivity-bounded networks. Using the homogeneity assumption in the case of SF networks will lead to a serious over-estimate of the epidemic threshold even for relatively small networks. 


\subsection{Immunization of scale-free networks}

As we have seen in Section 1.5, epidemic processes in SF networks do not possess, in the limit of an infinitely large network, an epidemic threshold below which diseases cannot set into an endemic state. SF networks are in this sense very prone to the spreading and persistence of infections, whatever virulence (parametrized by the spreading rate $\lambda$ ) the infective agent might possess. In view of this weakness, it becomes a major task to find optimal immunization strategies oriented to minimize the risk of epidemic outbreaks in SF networks.

\subsubsection{Uniform immunization}

The simplest immunization procedure one can consider consists in the random introduction of immune individuals in the population [3], in order to get a uniform immunization density. In this case, for a fixed spreading rate $\lambda$, the relevant control parameter in the density of immune nodes present in the network, the immunity $g$. At the mean-field level, the presence of a uniform immunity will have the effect of reducing the spreading rate $\lambda$ by a factor $1-g$; i.e. the probability of finding and infecting a susceptible and nonimmune node will be $\lambda(1-g)$. For homogeneous networks we can easily see that, for a constant $\lambda$, the stationary prevalence is given in this case by

$$
\begin{array}{ll}
\rho_{g}=0 & \text { if } g>g_{c}, \\
\rho_{g}=\left(g_{c}-g\right) /(1-g) & \text { if } g \leq g_{c},
\end{array}
$$

where $g_{c}$ is the critical immunization value above which the density of infected individuals in the stationary state is null and depends on $\lambda$ as

$$
g_{c}=1-\frac{\lambda_{c}}{\lambda}
$$

Thus, for a uniform immunization level larger than $g_{c}$, the network is completely protected and no large epidemic outbreaks are possible. On the contrary, uniform immunization strategies on SF networks are totally ineffective. The presence of uniform immunization is able to locally depress the infection's prevalence for any value of $\lambda$, but it does so too slowly, and it is impossible to find any critical fraction of immunized individuals that ensures the infection eradication. After a moment's reflection, one can convince oneself of the reason of this failure: With the uniform immunization strategy we are giving the same weight to very connected nodes (with the largest infection potential) and to nodes with a very small connectivity (which are relatively safe). Due to the large fluctuations in the connectivity, heavily connected nodes, which are statistically very significant, can overcome the effect of the immunization and maintain the endemic state. On the other hand, the absence of an epidemic threshold $\left(\lambda_{c}=0\right)$ in the thermodynamic limit implies that whatever rescaling $\lambda \rightarrow \lambda(1-g)$ of the spreading rate does not eradicate the infection except the case $g=1$. Indeed, by inserting Eq. (1.14) into Eq. (1.23) we have that the immunization threshold is given by

$$
1-g_{c}=\frac{1}{\lambda} \frac{\langle k\rangle}{\left\langle k^{2}\right\rangle} .
$$


In SF networks with $\left\langle k^{2}\right\rangle \rightarrow \infty$ only a complete immunization of the network (i.e. $g_{c}=1$ ) ensures an infection-free stationary state. The fact that uniform immunization strategies are less effective has been noted in several cases of spatial heterogeneity [3]. In SF networks we face a limiting case due to the extremely high (virtually infinite) heterogeneity in the connectivity properties. Specifically, it follows from Eq. (1.19) that the SIS model on the BA network shows for $g \simeq 1$ and any $\lambda$ the prevalence

$$
\rho_{g} \simeq 2 \exp [-1 / m \lambda(1-g)]
$$

In other words, the infection always reaches an endemic state if the network size is large enough (see Fig. 1.7 (a)). This fact points out the absence of an immunization threshold; SF networks are weak in face of infections, also after massive uniform vaccination campaigns.

\subsubsection{Targeted immunization}

We have seen in Section 1.6.1 that the very peculiar nature of SF networks hinders the efficiency of naive uniform immunization strategies. However, we can take advantage of the heterogeneity of SF networks, by devising an immunization strategy that takes into account the inherent hierarchy in the network's nodes. In fact, it has been shown that SF networks posses a noticeable resilience to random connection failures [40, 41, 42, which implies that the network can resist a high level of damage (disconnected links), without loosing its global connectivity properties; i.e. the possibility to find a connected path between almost any two nodes in the system. At the same time, SF networks are strongly affected by selective damage; if a few of the most connected nodes are removed, the network suffers a dramatic reduction of its ability to carry information [40, 41, 42]. Applying this argument to the case of epidemic spreading, we can devise a targeted immunization scheme in which we progressively make immune the most highly connected nodes, i.e., the ones more likely to spread the disease. While this strategy is the simplest solution to the optimal immunization problem in heterogeneous populations [ [3], its efficiency is comparable to the uniform strategies in homogeneous networks with finite connectivity variance. In SF networks, on the contrary, it produces an arresting increase of the network tolerance to infections at the price of a tiny fraction of immune individuals.

We can make an approximate calculation of the immunization threshold in the case of a random SF network [20]. Let us consider the situation in which a fraction $g$ of the individuals with the highest connectivity have been successfully immunized. This corresponds, in the limit of a large network, to the introduction an upper cut-off $k_{t}$-which is obviously an implicit function of the immunization $g-$, such that all nodes with connectivity $k>k_{t}$ are immune. The introduction of immune nodes implies at the same time the elimination of all the links emanating from them, which translates, in a mean-field approximation, into a probability $p(g)$ of deleting any link in the network. This elimination of links yields a new connectivity distribution, for which all moments can be computed. Recalling Eq. (1.14), we can then compute the critical fraction $g_{c}$ of immune individuals needed to eradicate the infection. An explicit calculation for the BA network [20] yields the approximate solution for the immunization threshold in the case of targeted immunization as

$$
g_{c} \simeq \exp (-2 / m \lambda)
$$



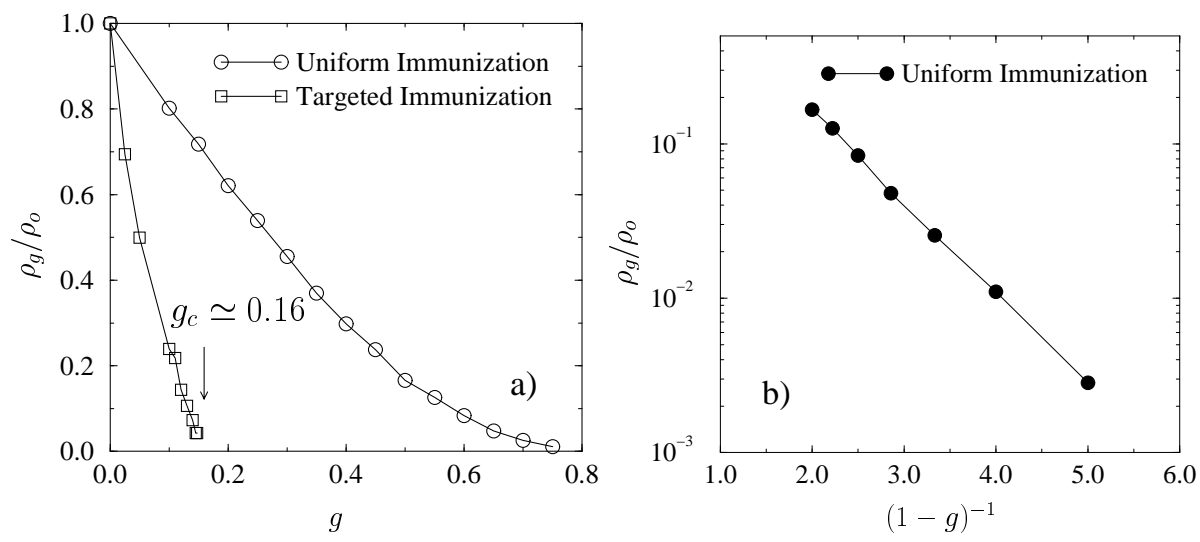

Figure 1.7: a) Reduced prevalence $\rho_{g} / \rho_{0}$ from computer simulations of the SIS model in the BA network with uniform and targeted immunization, at a fixed spreading rate $\lambda=0.25$. A linear extrapolation from the largest values of $g$ yields an estimate of the threshold $g_{c} \simeq 0.16$ in BA networks with targeted immunization. b) Check of the predicted functional dependence $\rho_{g} \sim \exp (-1 / m \lambda(1-g))$ for the SIS model in the BA network with uniform immunization.

This clearly indicates that the targeted immunization program is extremely convenient in SF networks where the critical immunization is exponentially small in a wide range of spreading rates $\lambda$.

In order to assess the efficiency of the targeted immunization scheme we show in Fig. 1.7 the results from numerical simulations of the SIS model on BA networks, together with the results from simulations with uniform immunization [20]. In particular the plot shows the reduced prevalence $\rho_{g} / \rho_{0}$, where $\rho_{0}$ is the prevalence in the nonimmunized network, as a function of the fraction of immunized nodes $g$, at a fixed spreading rate $\lambda=0.25$. Fig. 1.7(a) indicates that, for uniform immunization, the prevalence decays slowly when increasing $g$, and will be effectively null only for $g=1$, as predicted by Eq. (1.25). In fact, the plot in Fig. 1.7 (b) recovers the theoretical predicted behavior Eq. 1.25). On the other hand, for the targeted immunization, the prevalence shows a very sharp drop and exhibits the onset of an immunization threshold above which the system in infection-free. A linear regression from the largest values of $g$ yields an approximate estimation $g_{c} \simeq 0.16$, that definitely proves that SF networks are very sensitive to the targeted immunization of a very small fraction of the most connected nodes.

This result can be readily extended to SF networks with arbitrary $\gamma$ values, and it is also possible to devise alternative immunization schemes which take advantage of the SF connectivity patterns in order to achieve a high level of tolerance to infections [20]. Other strategies have been put forward in Ref. [21] by proposing to cure with proportionally higher rates the most connected nodes. Also in this case it is possible to reintroduce a threshold in the network with this hub-biased policy for the administered cures.

The present results indicate that the SF networks' susceptibility to epidemic spreading is reflected also in an intrinsic difficulty in protecting them with local—uniform-immunization. 
On a global level, uniform immunization policies are not satisfactory and only targeted immunization strategies successfully lower the vulnerability of SF networks. This evidence radically changes the usual perspective of the regular epidemiological framework. Spreading of infectious or polluting agents on SF networks, such as food or social webs, might be contrasted only by a careful choice of the immunization procedure. In particular, these procedures should rely on the identification of the most connected individuals. The protection of just a tiny fraction of these individuals raises dramatically the tolerance to infections of the whole population. The computer virus case is once again providing support to this picture. Despite deployment of antivirus software is timely and capillary, viruses' lifetimes are extremely long; in other words, very high levels of immunization are not able to eradicate the epidemic. In the standard epidemic framework this would be possible only in the case of very high spreading rate for the virus that is in contradiction with the always small prevalence of epidemic outbreaks. These empirical findings are, however, in good agreement with the picture obtained for the immunization of SF networks. In fact, the antivirus deployment is not eradicating the epidemics on the global scale since it is alike to a random immunization process where file scanning and antivirus updating are statistically left to the good will of users and system managers. Needless to say, from the point of view of the single user, antiviruses are extremely important, being the only way to ensure local protection for the computer.

\subsection{Conclusions}

The topology of the network has a great influence in the overall behavior of epidemic spreading. The connectivity fluctuations of the network play a major role by strongly enhancing the infection's incidence. This issue assumes a particular relevance in the case of SF networks that exhibit connectivity fluctuations diverging with the increasing size $N$ of the web. Here we have reviewed the new epidemiological framework obtained in population networks characterized by a scale-free connectivity pattern. SF networks are very weak in face of infections, presenting an effective epidemic threshold that is vanishing in the limit $N \rightarrow \infty$. In an infinite population this corresponds to the absence of any epidemic threshold below which major epidemic outbreaks are impossible. SF networks' susceptibility to epidemic spreading is reflected also in an intrinsic difficulty in protecting them with local-uniform-immunization policies. Only targeted immunization procedures achieve the desired lowering of epidemic outbreaks and prevalence.

The present picture qualitatively fits the observations from real data of computer virus spreading, and could solve the long standing problem of the generalized low prevalence and long lifetime of computer viruses without assuming any global tuning of the spreading rates. Moreover, recent findings on the web of human sexual contacts [12] prompt that the presented results could have potentially interesting implications also in the case of human sexual disease control.

In order to illustrate the new features of epidemic spreading in SF networks, we used the SIS model. It is important to stress, however, that the analysis on SF networks of different models, such as the SIR model, confirm the presented epidemiological picture [17, 18, 19]. Yet, many other ingredients concerning the infection mechanisms should be considered in a more realistic representation of real epidemics [3, 2]. In addition, simple rules defining the 
temporal patterns of the networks, such as the frequency of forming new connections, the actual time that a connection exists, or different types of connections, should be included in the modeling. These dynamical features are highly valuable experimental inputs which are necessary ingredients in the use of complex networks theory in epidemic modeling.

\section{Acknowledgements}

This work has been partially supported by the European Network Contract No. ERBFMRXCT980183 and by the European Commission - Fet Open project COSIN IST-2001-33555. R.P.-S. acknowledges financial support from the Ministerio de Ciencia y Tecnología (Spain).

\section{References}

[1] N. T. J. Bailey, The mathematical theory of infectious diseases, (Griffin, London, 1975). 2nd edition.

[2] O. Diekmann and J.A.P Heesterbeek, Mathematical epidemiology of infectious diseases: model building, analysis and interpretation, (John Wiley \& Sons, New York, 2000).

[3] R. M. Anderson and R. M. May, Infectious diseases in humans, (Oxford University Press, Oxford, 1992).

[4] A.-L. Barabási and R. Albert. Emergence of scaling in random networks. Science 286, 509-511 (1999).

[5] S. N. Dorogovtsev, J.F.F. Mendes, and A. N. Samukhin. Structure of growing networks with preferential linking. Phys. Rev. Lett. 85, 4633-4636 (2000).

[6] R. Albert and A.-L. Barabási. Statistical mechanics of complex networks. Rev. Mod. Phys. 74, 47-97 (2002).

[7] M. Faloutsos, P. Faloutsos, and C. Faloutsos. On power-law relationship of the Internet topology. Comput. Commun. Rev. 29, 251-263 (1999).

[8] G. Caldarelli, R. Marchetti, and L. Pietronero. The fractal properties of Internet. Europhys. Lett. 52, 386 (2000).

[9] R. Pastor-Satorras, A. Vázquez, and A. Vespignani. Dynamical and correlation properties of the Internet. Phys. Rev. Lett. 87, 258701 (2001).

[10] S.-H. Yook, H. Jeong, and A.-L. Barabási. Modeling the Internet's large-scale topology, (2001). e-print cond-mat/0107417.

[11] K.-I. Goh, B. Kahng, and D. Kim. Fluctuation-driven dynamics of the internet topology. Phys. Rev. Lett 88, 108701 (2002).

[12] F. Liljeros, C. R. Edling, L. A. N. Amaral, H. E. Stanley, and Y. Aberg. The web of human sexual contacts. Nature 411, 907-908 (2001).

[13] G. Abramson and M. Kuperman. Small world effect in an epidemiological model. Phys. Rev. Lett. 86, 2909-2912 (2001).

[14] C. Moore and M. E. J. Newman. Epidemics and percolation in small-world networks. Phys. Rev. E 61, 5678 (2000). 
[15] R. Pastor-Satorras and A. Vespignani. Epidemic spreading in scale-free networks. Phys. Rev. Lett. 86, 3200-3203 (2001).

[16] R. Pastor-Satorras and A. Vespignani. Epidemic dynamics and endemic states in complex networks. Phys. Rev. E 63, 066117 (2001).

[17] R. M. May and A. L. Lloyd. Infection dynamics on scale-free networks. Phys. Rev. E 64, 066112 (2001).

[18] Y. Moreno, R. Pastor-Satorras, and A. Vespignani. Epidemic outbreaks in complex heterogeneous networks. Eur. Phys. J. B 26, 521-529 (2002).

[19] M. E. J. Newman. Exact solutions of epidemic models on networks, (2001). e-print cond-mat/0201433.

[20] R. Pastor-Satorras and A. Vespignani. Immunization of complex networks. Phys. Rev. E 65, 036104 (2001).

[21] Z. Dezsö and A.-L. Barabási. Halting viruses in scale-free networks, (2001). e-print cond-mat/0107420.

[22] S. M. Bellovin. Packets found on an Internet. Comput. Commun. Rev. 23, $26-31$ (1993).

[23] C. D. Harley, R. Slade, D. Harley, E. H. Spafford, and U E. Gattiker, Viruses Revealed, (McGraw-Hill, New York, 2001).

[24] J. O. Kephart, G. B. Sorkin, D. M. Chess, and S. R. White. Fighting computer viruses. Scientific American 277(5), 56-61 November (1997).

[25] J. O. Kephart and S. R. White. Directed-graph epidemiological models of computer viruses. In Proceedings of the 1991 IEEE computer society symposium on research in security and privacy (SSP '91), 343-361 (IEEE, Washington - Brussels - Tokyo, 1991).

[26] J. O. Kephart, S. R. White, and D. M. Chess. Computers and epidemiology. IEEE Spectrum 30, 20-26 (1993).

[27] G. Chartrand and L. Lesniak, Graphs \& digraphs, (Wadsworth \& Brooks/Cole, Menlo Park, 1986).

[28] P. Erdös and P. Rényi. On the evolution of random graphs. Publ. Math. Inst. Hung. Acad. Sci. 5, 17-60 (1960).

[29] D. J. Watts and S. H. Strogatz. Collective dynamics of 'small-world' networks. Nature 393, 440-442 (1998).

[30] J. Marro and R. Dickman, Nonequilibrium phase transitions in lattice models, (Cambridge University Press, Cambridge, 1999).

[31] J. O. Kephart and S. R. White. Measuring and modeling computer virus prevalence. In Proceedings of the 1993 IEEE computer society symposium on security and privacy (SSP '93), 2-15 (IEEE, Washington - Brussels - Tokyo, 1993).

[32] S. R. White. Open problems in computer virus research. Virus Bulletin Conference, Munich, October (1998). Available on-line at http://www.av.ibm.com/ScientificPapers/White/Problems/Problems.html.

[33] R. Albert, H. Jeong, and A.-L. Barabási. Diameter of the world-wide web. Nature 401, 130-131 (1999).

[34] S. Wasserman and K. Faust, Social network analysis, (Cambridge University Press, Cambridge, 1994). 
[35] H. Ebel, L.-I. Mielsch, and S. Bornholdt. Scale-free topology of e-mail networks, (2002). e-print cond-mat/0201476.

[36] A.-L. Barabási, R. Albert, and H. Jeong. Mean-field theory for scale-free random networks. Physica A 272, 173-187 (1999).

[37] L. A. N. Amaral, A. Scala, M. Barthélémy, and H. E. Stanley. Classes of small-world networks. Proc. Natl. Acad. Sci. USA 97, 11149-11152 (2000).

[38] S. N. Dorogovtsev and J. F. F. Mendes. Evolution of networks. Advances in Physics 51, 1079-1187 (2002).

[39] R. Pastor-Satorras and A. Vespignani. Epidemic dynamics in finite size scale-free networks. Phys. Rev. E 65, 035108 (2002).

[40] R. A. Albert, H. Jeong, and A.-L. Barabási. Error and attack tolerance of complex networks. Nature 406, 378-382 (2000).

[41] D. S. Callaway, M. E. J. Newman, S. H. Strogatz, and D. J. Watts. Network robustness and fragility: percolation on random graphs. Phys. Rev. Lett. 85, 5468-5471 (2000).

[42] R. Cohen, K. Erez, D. ben Avraham, and S. Havlin. Breakdown of the Internet under intentional attack. Phys. Rev. Lett. 86, 3682-3685 (2001). 